\title{
Notizie dall'Accademia
}

\section{A cura di comitato di redazione}

PUBBLICATO: 30 GIUGNO 2021

$\mathrm{D}$ ue sono le Tornate accademiche che sono state organizzate durante la primavera del 2O2I. La prima, dedicata a Giorgio Petrocchi (Codici, facsimili ed edizioni nell'anno di Dante. In memoria di Giorgio Petrocchi, a 100 anni dalla nascita, mercoledi 26 maggio 202I), ha beneficiato dei contributi di Giovanna Frosini, Marcello Ciccuto, Giorgio Inglese, Francesca Petrocchi e Barbara Bertoni; la seconda, dedicata a Dante nell'anno in cui si celebrano i 700 anni dalla sua morte (Non solo italiano Dante, il "De vulgari eloquentia" e le lingue: una lezione per l'Europa?, martedi I5 giugno 202I), ha visto invece susseguirsi gli interventi di Mirko Tavoni, Irène Rosier-Catach, Vittorio Coletti, Francesco Sabatini. Entrambi gli eventi erano presieduti dal presidente dell'Accademia, Claudio Marazzini, si sono svolti in modalità telematica ed erano aperti a tutti gli interessati.

Ha beneficiato dell'organizzazione da remoto anche l'incontro di Lessicografia tenuto lunedi is aprile dall'Accademia Brasiliana di Lettere. L'obiettivo era quello di coinvolgere alcune delle Accademie "gemelle" che nel mondo si occupano di lingue neolatine: tra queste naturalmente figurava anche l'Accademia della Crusca, per la quale ha partecipato Claudio Marazzini.

Il presidente è intervenuto anche in occasione del Festival della Luce (Como, I5-23 maggio), organizzato dalla Fondazione Alessandro Volta e quest'anno dedicato, oltre che ai consueti temi scientifico-divulgativi, anche a Dante. Lintervento del presidente si è svolto durante la serata del i5 maggio intitolata Dal Buio alla Luce: il Viaggio dantesco e la Cosmologia Moderna ed ha avuto come soggetto La luce nella lingua italiana.

Svoltisi ancora in modalità virtuale, ma sulle pagine social dell'Accademia, due incontri a tema linguistico che hanno coinvolto ospiti popolari al grande pubblico: il primo, con la presidente onoraria Nicoletta Maraschio e Michela Murgia, era dedicato al linguaggio di genere e alla presentazione dell'ultimo libro della scrittrice (Michela Murgia in dialogo con Nicoletta Maraschio, 8 aprile); il secondo, al quale hanno partecipato Giuseppe Patota, accademico, Marco Biffi, responsabile di molti progetti promossi dall'Accademia e del suo sito web e il cantuatore Lorenzo Baglioni, ha visto i partecipanti dialogare sul rapporto tra norma e uso linguistico (Piuttosto che: istruzioni per l'uso, II maggio).

La presidente onoraria dell'Accademia, che da anni fa parte della giuria che conferisce il Premio Strega, è stata coinvolta insieme a Raffaella Setti, collaboratrice della "Crusca per Voi" e della redazione Consulenza linguistica della Crusca, nel convegno online Strega 75. Narrazioni e visioni dal dopoguerra a oggi (8 giugno 202I), durante il quale si è ripercorsa la storia del Premio e si è discusso il suo rapporto con il cinema.

Di grande interesse per l'Accademia è stato il seminario Per un museo della lingua italiana, incentrato, appunto, sul progetto a lungo caldeggiato dalla Crusca e da molti linguisti e storici italiani di uno spazio espositivo multimediale dedicato alla lingua, alla sua storia e alla sua evoluzione, progetto ormai in fase di realizzazione a Firenze. Il seminario, tenutosi in rete venerdi 28 maggio, ha rappresentato un'altra tappa importante verso il consolidamento dell'idea dell'esposizione. In rappresentanza della Crusca, l'accademica Rita Librandi ha presieduto una sessione del dibattito. 
Presso la Biblioteca del Comune di Vinci (Firenze) è stato possibile discutere di un altro progetto che coinvolge l'Accademia, E-Leo 2.o: un archivio digitale contenente il patrimonio di manoscritti e disegni di Leonardo da Vinci, realizzato dalla Biblioteca leonardiana di Vinci con la collaborazione dell'Accademia della Crusca e l'Università di Firenze (E-Leo 2.o. L'archivio digitale di storia della tecnica e della scienza della Biblioteca leonardiana di Vinci, giovedi I7 giugno 202I). Tra i relatori era presente, in rappresentanza della Crusca, Marco Biffi.

Ugualmente dedicato alla lingua di Leonardo è stato l'incontro Ancora su Leonardo nomo di lettere. Esplorazioni linguistiche (I8 maggio 202I), organizzato dal Centro di Studi sul Classicismo di Prato e tenuto dall'accademica Paola Manni.

Ricordiamo volentieri anche la presenza della fortunata iniziativa La parola di Dante fresca di giornata all'interno di una mostra dedicata alla figura e all'opera di Dante Alighieri organizzata dalla Galleria Rhinoceros e dalla Fondazione Alda Fendi di Roma. La scelta dei curatori di inserire il progetto dell'Accademia all'interno del proprio percorso espositivo è solo uno dei segnali del grande successo riscosso dalla Parola di Dante fresca di giornata, appuntamento digitale giornaliero di tutto l'anno dantesco.

Segnaliamo infine due iniziative dedicate alla formazione che in questa primavera hanno toccato l'Accademia: lo svolgimento della X edizione delle Olimpiadi di Italiano, la cui finale nazionale ha avuto luogo martedi i maggio coinvolgendo diversi accademici (il presidente Marazzini, Valeria Della Valle, Giovanna Frosini, Carla Marello, Luca Serianni), e quello del Corso di perfezionamento in Professioni legali e scrittura del diritto. Tecniche di redazione per atti chiari e sintetici (VII edizione, a partire dal i aprile). Il corso è organizzato dai Dipartimento di Scienze Giuridiche (DSG) e dal Dipartimento di Lettere e Filosofia dell'Università di Firenze (DILEF) in collaborazione con l'Accademia della Crusca, la Scuola Superiore della Magistratura, Informatica Giuridica e Sistemi Giudiziari (IGSG), la Camera Civile di Firenze, l'Ordine degli avvocati di Firenze, la Fondazione per la formazione forense dell'Ordine degli avvocati di Firenze, la Ordine degli avvocati di Trento. Come ogni anno, il corso si svolge nell'arco di una serie di incontri tenuti da giuristi e linguisti, tra cui alcuni accademici e collaboratori dell'Accademia: Federigo Bambi, Marco Biffi, Michele Cortelazzo, Riccardo Gualdo, Cecilia Robustelli, Angela Frati e Stefania Iannizzotto.

Ricordiamo infine un avvenimento doloroso: la scomparsa del linguista Ivan Kajn. Di nazionalità serba, Kajn aveva insegnato presso l'Università di Belgrado ed era accademico corrispondente della Crusca dal settembre 20I3.

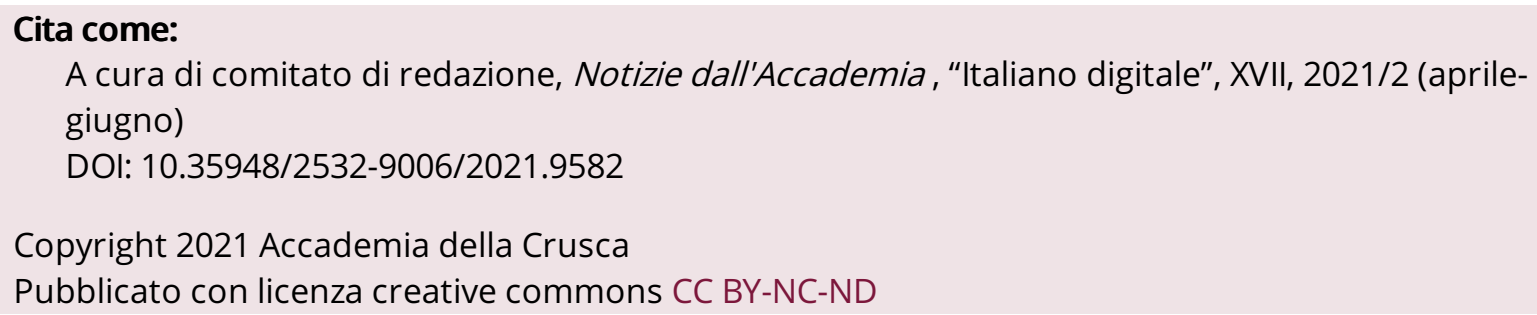

